\title{
Diagnosing the Excitation Mechanisms of Pc5 Magnetospheric Flank Waveguide Modes and FLRs
}

\author{
Ian R. Mann \\ Department of Physics, University of York, Heslington, York, YO10 5DD, U.K.
}

\author{
Andrew. N. Wright \\ Mathematical Institute, University of St. Andrews, St. Andrews, Fife, KY16 9SS, Scotland.
}

\begin{abstract}
Using the theoretical results of Mann et al. [1999], we show how the characteristics of multiple field line resonances (FLRs), driven by waveguide mode harmonics, can be strongly local time dependent and how these characteristics can be used to differentiate between waves driven by solar wind stimuli or by the development of magnetopause shear flow instabilities. Using observations previously reported in the literature, we show examples of this diagnostic analysis and conclude that the dawn/dusk asymmetry in Pc5 FLR characteristics may be linked to different dominant excitation mechanisms operating either side of noon. In particular, we suggest there is a preference for dawn-side FLRs to be excited by waveguide modes which are amplified through the action of over-reflection at the magnetopause.
\end{abstract}

\section{Introduction}

Recent work has considered the excitation of field line resonances (FLRs) by magnetospheric waveguide modes [e.g., Walker et al., 1992; Wright, 1994]. The waveguide modes stand between an outer magnetospheric boundary (usually the magnetopause) and an inner turning point, and hence have a discrete (i.e., quantized) frequency spectrum; FLRs being excited at the harmonic frequencies of the waveguide. Waveguide modes can be driven by solar wind discontinuities [c.f., Rostoker and Sullivan, 1987], by continual random buffeting [e.g., Smith et al., 1998], through the growth of unstable KH waves [e.g., Fujita et al., 1996], or through the over-reflection of body waveguide modes at an unstable magnetopause [Walker, 1998; Mann et al., 1999; Mills et al., 1999]. In this letter we use the theory developed by Mann et al. [1999] (hereafter MWMN) and Mills et al. [1999] to address (1) the local time and solar wind conditions under which differing sources can act as efficient drivers of waveguide modes, (2) the likely characteristics of any FLRs driven by the waveguide modes, and (3) examine diagnostics which can distinguish between sources.

\section{Magnetopause Boundary Conditions.}

In previous studies the magnetopause bounding the waveguide is usually assumed to be fixed and perfectly reflecting [e.g., Allan et al., 1986]. In a recent paper, however, MWMN considered the modes supported by a uniform waveguide with a free magnetopause boundary which separates a stationary cold magnetosphere (with Alfvén speed $c_{A}$ ) from a flowing warm and field free magnetosheath (with sound speed $c_{s}$ ), which should be a reasonable approximation to reality [see also Fujita et al., 1996]. MWMN found that for the magnetospheric case (where $c_{s} / c_{A}<1$ ) and for slow

Copyright 1999 by the American Geophysical Union.

Paper number 1999GL900573.

0094-8276/99/1999GL900573\$05.00 magnetosheath flow speeds the magnetopause was always imperfectly reflecting. Hence near the sub-solar point, where the magnetosheath flow speed $U$ is small, waveguide mode energy will always leak out across the magnetopause. Only at local times where $U$ becomes sufficiently fast does the magnetopause become perfectly reflecting. Of more significance was MWMN's examination of the growth of magnetopause instabilities. Their solutions included not only the usual Kelvin-Helmholtz (KH) surface waves, but also growing $(\mathrm{KH})$ waveguide mode solutions which could be excited at local times where the magnetosheath flow speed $U>c_{A}+c_{s}$ through over-reflection and the propagation of negative energy waves in the magnetosheath.

These three magnetopause boundary conditions translate to different magnetospheric local times. Figure 1a shows this schematically for moderate solar wind speed. In this case, the near-nose magnetopause is leaky and only able to support low quality $(Q)$ and hence broadband (in frequency) waveguide mode harmonics. These leaky low $Q$ waveguide modes would not normally be expected to drive clear resonance signatures, any Alfvén waves which are excited will probably be driven over a broad range of L-shells and be relatively small amplitude. Towards the dawn and dusk flanks, however, the magnetopause boundary may become perfectly reflecting at some local time. The waveguide modes excited beyond this point will have much higher $Q$, a narrower frequency bandwidth, be longer-lived, and can hence act as coherent drivers of clear FLRs. For faster solar wind speeds, the transition to a perfectly reflecting magnetopause for a particular frequency waveguide mode will occur closer to local noon. For sufficiently fast flows and for waveguide modes with sufficiently low frequencies, a region of over-reflection will develop on the flanks, as illustrated in Figure $1 \mathrm{~b}$. Higher frequency waveguide modes will only be excited by over-reflection if the solar wind speed is large.

The over-reflection model of MWMN predicts both the quantisation of mode frequencies and the preferential excitation of FLRs on the flanks. Over-reflection should occur when $U>c_{A}+c_{s}$ (stabilising magnetosheath field line tension is neglected in the MWMN model), and using typical values for $c_{A}$ and $c_{s}, U$ needs to be $\gtrsim 500 \mathrm{kms}^{-1}$. This is in excellent agreement with the recent observations of Engebretson et al. [1998] who found a cutoff of Pc5 power on the dawn flank for $v_{S W} \lesssim 500 \mathrm{kms}^{-1}$. Observations have tended to show a prevalence of FLRs at dawn compared to dusk. This may be related to dawn/dusk asymmetry in magnetopause stability and we discuss this in section 4 .

\section{Diagnosing Excitation Mechanisms.}

\subsection{Theory}

Wright and Rickard [1995] showed that comparing the phase speeds of multiple FLRs driven by waveguide mode harmonics can be used as a diagnostic to distinguish between waveguide mode energy sources. In this letter the diagnostic is extended and applied to the more realistic case of a free 

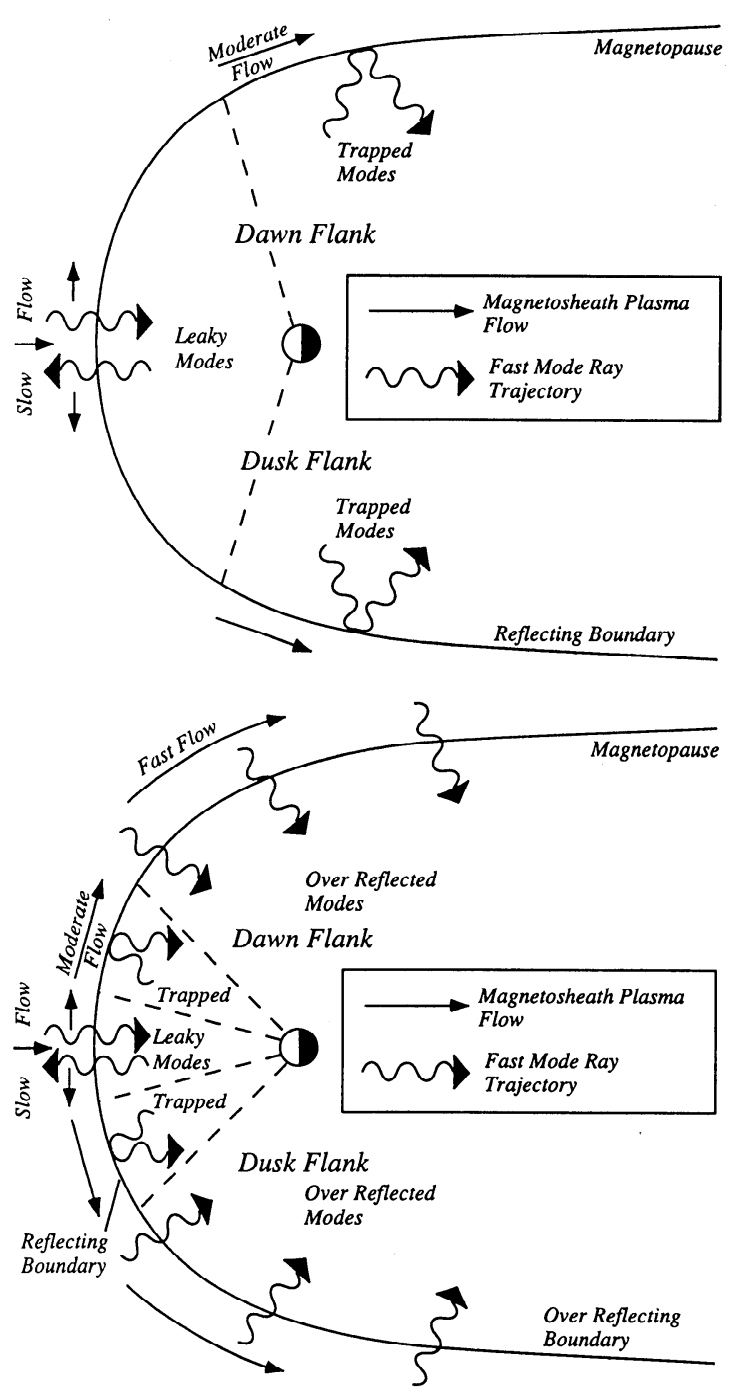

Figure 1. Schematic of waveguide mode magnetopause boundary conditions for (a) moderate solar wind speed, and (b) high solar wind speed.

magnetopause boundary considered by MWMN. The flow chart in Figure 2 summarises our results. To use the chart requires the measurement of the azimuthal phase velocities of two FLRs that occured simultaneously and at a similar local time. Each phase speed $\left(v_{p h}\right)$ must be mapped to the magnetopause to get the corresponding azimuthal phase speed there $\left(v_{p h M P}\right)$. Theoretical models and the discussion below often use a cartesian geometry where $y$ represents the azimuthal coordinate and $k_{y}$ the azimuthal wavenumber.

If the two values derived for $v_{p h M P}$ are different, Figure 2 indicates that the FLRs could have been driven by impulsive buffeting (hereafter IB) of the magnetopause, in accordance with Wright and Rickard [1995]. They showed that the azimuthal wavenumbers of such IB FLRs are determined by the details of the magnetospheric equilibrium, each of the multiple IB FLRs being excited with different $v_{p h} ; \omega_{r}$ is determined from the fast mode dispersion relation with $k_{y} \approx 0$ (see Walker et al. [1992] and Wright [1994]). It is also possible that a variety of different excitation mechanisms could be operating. (see below).

If the FLRs have a common $v_{p h M P}$, but $U<c_{A}+c_{s}$, over-reflection can not operate, so the FLRs must be driven a "running pulse" (hereafter RP) on the magnetopause, such as the contact point of a shock or density discontinuity. The R.P driver was investigated by Wright and Rickard [1995], who found that $v_{p h M P}$ was equal to $U_{b}$ (the speed of the RP along the magnetopause). The FLR azimuthal wavenumber $k_{y}$ is calculated from $v_{p h}=\omega_{r} / k_{y}$ with $\omega_{r}$ determined from the fast mode dispersion relation with $k_{y} \approx 0$.

For FLRs with a common $v_{p h M P}$ and $U>c_{A}+c_{s}$, overreflection can operate. If $v_{p h M P}>U-c_{s}$ these modes will not experience over-reflection, and their common phase speed is probably due to RP driving. The FLRs will have frequencies and wavenumbers as described in the previous paragraph. For FLRs with a common $v_{p h M P}$, a magnetopause capable of over-reflection $\left(U>c_{A}+c_{s}\right)$, and a phase speed meeting the over-reflection criterion $\left(v_{p h M P}<\right.$ $\left.U-c_{s}\right)$, the FLRs are most likely driven by the overreflection mechanism (hereafter OR). MWMN suggest that waveguide modes driven by OR will have preferred $k_{y}$ s corresponding to the waveguide modes with the fastest growth rates. Moreover, an FLR and the OR waveguide mode driving it will have the same $k_{y}$ and $\omega_{r}$. Mills and Wright [1999] showed that the phase velocities of each of these fastest growing waveguide mode harmonics are all approximately the same. This is to be expected since over-reflection relies upon the growth of local magnetopause instabilities. A consequence of this is that OR waveguide modes and the FLRs they excite will have a common $v_{p h M P}$.

Note that it is not contradictory to have more than one FLR excitation mechanism operating simultaneously. For example, a localised impulsive disturbance at the magnetopause followed by an undriven period can be described (during the latter phase) as a sum of the normal modes of the system. For example, the lowest frequency harmonics could well be over-reflected, and following their initial seed perturbation could gain further energy from the magnetosheath flow. Higher harmonic modes might be driven through the RP or IB mechanisms, and these could excite further FLRs if the impulse was sufficiently large. These additional FLRs would have different $v_{p h}$ to the OR modes, their amplitudes depending in part upon whether the waveguide modes driving them were perfectly reflected at the magnetopause at the local time of the FLRs. In this situation the solar wind impulse might act as a driver for FLRs directly through the IB/RP mechanisms, but could also act as a seed perturbation for the growth of unstable waveguide modes (OR mechanism).

The ideas described above focus on FLRs driven by waveguide modes, not surface modes. Indeed, Kivelson and Southwood [1985] provide convincing arguments for cavity modes (i.e., modes that propagate in the outer magnetosphere) being better than surface modes at driving FLRs with discrete frequencies, and numerical simulations [e.g., Allan et al., 1986] support this. Moreover, whilst a realistic finite-thickness magnetopause can support a fastest growing $\mathrm{KH}$ surface wave with a preferred $k_{y}$, only one such mode exists for any given $U$. Only cavity-like modes, whose frequencies are structured by the natural frequencies of the waveguide, can produce the harmonic spectrum which is observed. This suggests body waveguide modes are ordinarily the drivers of Pc5 FLRs in the magnetosphere.

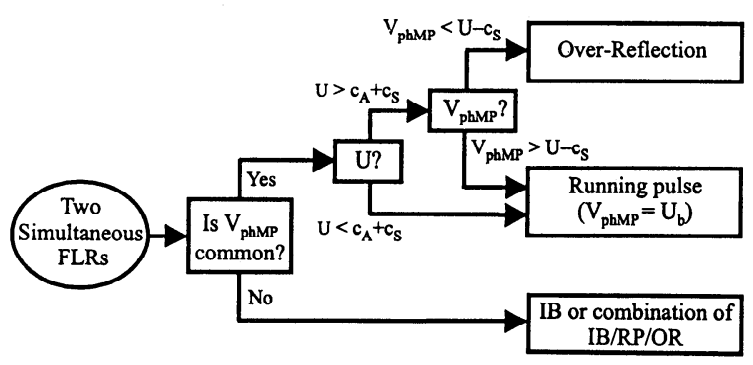

Figure 2. Flow chart determining the likely energy source of pairs of multiple harmonic FLRs. (IB, RP, and OR, represent impulsive buffeting, running pulse, and over-reflection sources respectively. See text for more details.) 


\subsection{Observations.}

We considered observations reported in the literature of the characteristics of multiple low- $m$ FLRs observed at the same time and with the same direction of propagation. We identified 10 events, 6 observed by radars (from Fenrich et al. [1995] and Fenrich [1997], referred to as A and B, respectively) and 4 by ground-based magnetometers (from Ziesolleck and McDiarmid [1994] and Ziesolleck and McDiarmid [1995], referred to as C and D, respectively). Whether the FLRs had a common phase velocity was determined in each case using the quoted errors in the estimated $m$ values. In cases where the error bars of one FLR phase velocity overlapped with the central estimate of another FLR phase velocity, the phase speed was deemed common. Cases where the overall error bars overlapped were deemed possibly common, whilst cases where there was no possible phase speed overlap within the error bars were deemed uncommon. Of the six events which were observed in the morning sector, five definitely had common phase velocities. In contrast, none of the 4 events which occurred near dusk definitely had common phase velocity. This suggests that, on average, different dominant waveguide mode excitation mechanisms might be operating at dawn and dusk.

To illustrate the diagnostic developed in Figure 2, we present in table 1 the subset of these events for which insitu solar wind speed measurements are available. Ideally we need satellite data providing $U, c_{s}$, and $c_{A}$ at the local time of the FLR, however we do not have this data for these events. This is particularly important for deciding whether FLRs with $v_{p h M P}<U-c_{s}$ were driven by the OR mechanism, or were the result of IB exciting low $v_{p h}$ FLRs; OR modes require $U>c_{A}+c_{s}$. Since the FLRs are all approximately on the dawn or dusk flank we make the crude assumption that $U \approx v_{S W}$, although we note that depending upon local time $U$ could be as low as $v_{S W} / 2$ on occasions.

Interestingly, all of the dawn flank FLR events in Table $1 \mathrm{had}$ common phase speeds and were identified as having probably been driven by OR. In contrast, none of the four dusk flank events had common phase speeds within our definition, however, one event possibly did (B.13e-f) and one

Table 1. Solar wind speeds $v_{S W}\left(\mathrm{kms}^{-1}\right)$ at the time of FLR observations. $v_{p h M P}\left(\mathrm{kms}^{-1}\right)$ is the FLR phase speed of each harmonic mapped to an assumed magnetopause located at $L=12$. The entries in brackets after $v_{p h M P}$ indicate whether the FLRs did not (entry N), possibly did (entry ?), or did (entry $\mathrm{Y}$ ) share a common $v_{p h M P}$ with another simultaneous FLR. Superscript* and superscript\# represent observations by Geotail and IMP8 respectively. Entry "any" represents a source of one of IB/RP/OR.

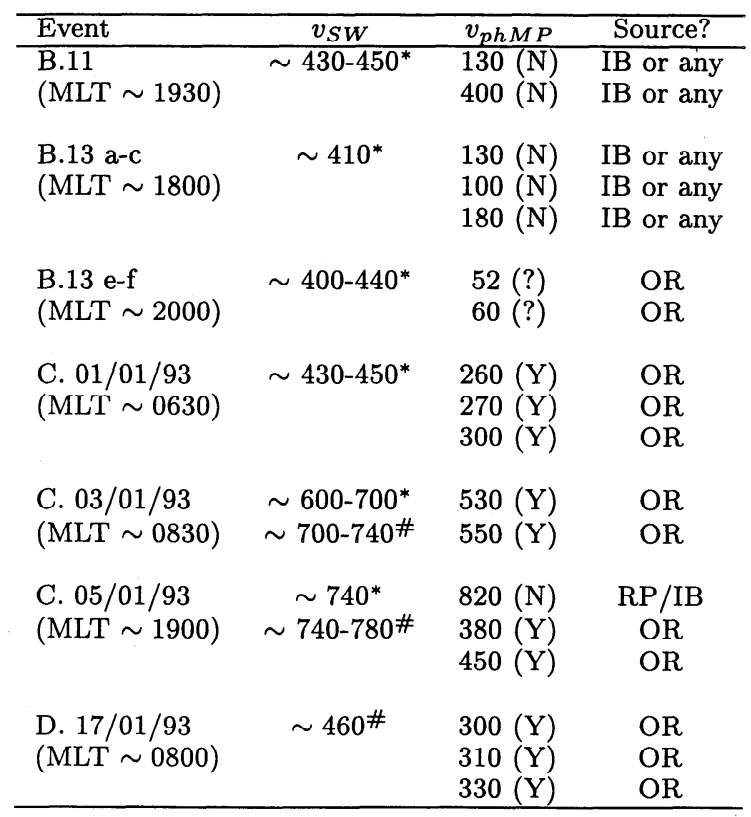

pair of the three FLRs identified in the C.05/01/93 event had common phase speeds. Both of these pairs of dusk FLR harmonics could have been driven by $O R$ and we discuss them further below. All of the events identified as OR seem to satisfy the condition $v_{p h M P}<U-c_{s}$, and based on the range of typical speeds observed inside and outside the magnetopause [e.g., Eastman et al., 1985] could have satisfied the condition $U>c_{A}+c_{s}$ required for over-reflection. In-situ magnetopause data for $c_{A}$ and $c_{s}$ would be needed to verify this assertion. Interestingly, counting the numbers of pairs of OR FLR harmonics we find that there are 7 of these pairs at dawn, and only 2 at dusk. Again, this indicates the tendancy for there to be an asymmetry in FLR characteristics across noon.

The dusk flank events B.13e-f and C.05/01/93 displayed pairs of FLRs which could have had common phase velocities. The phase velocities for all three of the C.05/01/93 FLR harmonics were interpretted as common by Ziesolleck and McDiarmid [1994] (see their figure 16), although closer inspection of their figure shows that they are not common within their error bars. The C.05/01/93 event does occur during a very high solar wind speed interval, so the condition $U>c_{A}+c_{s}$ could have been satisfied to make the late afternoon magnetopause over-reflecting. Two of the C.05/01/93 event FLRs have $v_{p h M P}=380 \mathrm{kms}^{-1}$ and $450 \mathrm{kms}^{-1}$ and these appear to satisfy the OR criteria $v_{p h M P}<U-c_{s}$, however, the $v_{p h M P}=820 \mathrm{kms}^{-1}$ FLR does not. This illustrates the value of our diagnostic, since we are able to infer that although two of the C.05/01/93 FLR harmonics were probably driven by OR, one was almost certainly not.

Similarly, the pair of FLR harmonics in the B.13e-f event were identified as probably being driven by OR $\left(v_{p h}\right.$ was possibly common), although $v_{S W}$ for this event was much smaller so that the criteria $U>c_{A}+c_{s}$ might not have been satisfied. If magnetic tension acts to stabilise the dusk flank magnetopause, then OR could have been even less likely. Inspection of the Geotail data from this day (not shown) show several large step decreases in solar wind density, including events around 0030 and $0100 \mathrm{UT}$, and several step changes in $|B|$, particularly notable changes occurring at around 0200 and 0230 UT. Each of these would have passed the Earth about 14 minutes earlier. On this day the Saskatoon radars saw three FLR wave packets around this time, including two with multiple harmonics (reported in Table 1) from 0030$0200 \mathrm{UT}$ (event B.13a-c) and 0230-0350UT (B.13e-f). It is likely that the excitation of the earlier harmonic FLRs was related to the density, and hence ram pressure, changes in the solar wind acting as an IB source, although two simultaneous IB/RP/OR sources could perhaps also have driven these waves. The latter FLRs, on the other hand, have a possibly common phase speed and could have been related to the step changes in incident magnetic pressure. Whilst it is possible that the B.13e-f FLRs could have been driven by a RP on the magnetopause, $v_{p h M P}$ is quite small which suggests that $\mathrm{OR}$ is a more likely candidate. An interesting possibility is that the discontinuity in $|B|$ could have provided an impulsive seed perturbation which could have excited waveguide modes. If at this later local time the magnetopause was unstable to $\mathrm{OR}$, this could have amplified two waveguide modes which subsequently drove the pair of common $v_{p h}$ FLRs.

One problem with the diagnostic scheme presented in figure 2 concerns the calculation of FLR azimuthal phase velocities $v_{p h}$ from radar and ground-magnetometer data. In general, radar observed FLRs tend to have phase velocities which are very much lower than those inferred by groundbased magnetometers [e.g., Ziesolleck et al., 1998]. The same trend is apparent in Table 1, with the dusk B.13e-f radar event (diagnosed as possibly driven by OR) having an average $v_{p h M P}$ of only $56 \mathrm{kms}^{-1}$ which is very much less than is inferred for the OR FLRs detected by the magnetometers. The reasons for this discrepancy are not clear, however if the radar phase velocities are accurate this suggests that the dawn side magnetopause may be unstable to over-reflection for almost the whole range of solar wind speeds incident upon the magnetosphere since the condition 
that $v_{p h M P}<U-c_{s}$ will be easier to satisfy (subject to suitably low magnetopause boundary $c_{A}$ and $c_{s}$ so that $U$ remains $\left.>c_{A}+c_{s}\right)$.

\section{Discussion.}

Pc5 pulsations show a clear dawn/dusk asymmetry in the magnetosphere. Pulsations appear to be much more prevalent at dawn than dusk, and the dawnside waves are much more likely to show clear classic field line resonance signatures [Rostoker and Sullivan, 1987; Ziesolleck and McDiarmid, 1995; Chisham and Orr, 1997]. Rostoker and Sullivan [1987] point out that the afternoon waves they report can be linked back to impulses in solar wind ram pressure in $75 \%$ of cases. Interestingly, Rostoker and Sullivan [1987] also notice that statistically resonance characteristics appear to recover in the afternoon after about 1600LT; a similar statistical demarkation line being found by Mathie et al. [1999] after about 1530LT. Both these papers suggest that the explanation may be due to the dawn flank being more unstable than the dusk flank (see Mathie et al. [1999] for more details).

Indeed, it is possible that the recovery of FLR characteristics in the late afternoon sector could be related to the development of OR magnetopause instabilities there; dusk side stabilising effects tending to generate magnetopause instabilities at later local times in the afternoon than the morning under the same solar wind conditions. If OR does occur preferentially at dawn compared to dusk, then the morphology of figure $1 \mathrm{~b}$ should be modified to include an asymmetry across noon in the local time position of the onset of overreflection. Future studies of FLRs at local times when data from magnetopause crossings are available would be desirable. If the magnetosheath flow speed $U$, and $c_{A}$ and $c_{s}$ just inside and outside the magnetopause were known at a local time with coincident ground-based FLR observations, the diagnostic scheme in figure 2 could be experimentally tested. Moreover, if the magnetosheath magnetic field were measured, the hypothesis that field line tension stabilisation causes the dawn/dusk asymmetry could be tested on a case study and statistical basis.

If the FLRs driven in the magnetosphere represent an admixture of waves driven by discontinuities in the solar wind and waves driven by the development of magnetopause instabilities, increased duskside magnetopause stability would result in the asymmetry discussed above. In this scenario, the majority of afternoon side waves might be driven by solar wind impulses, whilst morning waves could be driven by both OR magnetopause instabilities and by solar wind impulses. Indeed, if solar wind impulses occur only sporadically compared to dawnside magnetopause instability, the morningside waves might have characteristics dominated by waves driven by solar wind velocity shear, whilst afternoonside waves might be statistically dominated by more impulsive characteristics. Moreover, in this scenario, the dusk side waves would also occur less often than their dawn side counterparts, as has been previously reported [e.g., Rostoker and Sullivan, 1987]. This explanation appears to be consistent with the observed distribution of waves previously reported in the literature, and for the events considered in this letter.

Acknowledgments. I.R.M and A.N.W. are supported by U.K. PPARC Fellowships. K. Ackerson and L. Frank provided GEOTAIL CPI data. GEOTAIL magnetic field data were provided by S. Kokubun through DARTS at ISAS in Japan. IMP8 data were provided by the MIT Solar Wind Group via CDAWeb.

\section{References}

Allan, W., S. P. White, and E. M. Poulter, Impulse-excited hydromagnetic cavity and field-line resonances in the magnetosphere, Planet. Space Sci., 34, 371, 1986.

Chisham, G., and D. Orr, A statistical study of the local time asymmetry of Pc5 ULF wave characteristics observed at mid-latitudes by SAMNET, J. Geophys. Res., 102, 24,339, 1997.
Eastman, T. E., B. Popielawska, and L. A. Frank, Threedimensional plasma observations near the outer magnetospheric boundary, J. Geophys. Res., 90, 9519, 1985.

Engebretson, M., K.-H. Glassmeier, M. Stellmacher, W. J. Hughes, and H. Luhr, The dependence of high latitude Pc5 wave power on solar wind velocity and on the phase of high speed solar wind streams, J. Geophys. Res., In Press, 1998.

Fenrich, F. R., Field line resonance: Observation and theory, Ph.D. thesis, Dept. of Physics, University of Alberta, Edmonton, Canada, 1997.

Fenrich, F. R., J. C. Samson, G. Sofko, and R. A. Greenwald, ULF high- and low- $m$ field line resonances observed with the Super Dual Auroral Radar Network, J. Geophys. Res., 100, 21,535, 1995.

Fujita, S., K.-H. Glassmeier, and K. Kamide, MHD waves generated by the Kelvin-Helmholtz instability in a nonuniform magnetosphere, J. Geophys. Res., 101, 27,317, 1996.

Kivelson, M. G., and D. J. Southwood, Resonant ULF waves: A new interpretation, Geophys. Res. Lett., 12, 49, 1985.

Mann, I. R., A. N. Wright, K. J. Mills, and V. M. Nakariakov, Excitation of magnetospheric waveguide modes by magnetosheath flows, J. Geophys. Res., 104, 333, 1999.

Mathie, R. A., I. R. Mann, F. W. Menk, and D. Orr, Pc5 ULF pulsations associated with waveguide modes observed with the image magnetometer array, J. Geophys. Res., 104, 7025, 1999.

Mills, K. J., and A. N. Wright, Azimuthal phase speeds of FLRs driven by Kelvin-Helmholtz unstable waveguide modes, J. Geophys. Res., In Press, 1999.

Mills, K. J., A. N. Wright, I. R. Mann, and V. M. Nakariakov, Kelvin-Helmholtz driven modes of the magnetosphere, Phys. Plasmas, In Press, 1999.

Rostoker, G., and B. T. Sullivan, Polarisation characteristics of Pc5 magnetic pulsations in the dusk hemisphere, Planet. Space Sci., 35, 429, 1987.

Smith, J. M., A. N. Wright, and G. J. Rickard, ULF pulsations driven by a randomly varying magnetopause displacement, J. Geophys. Res., 103, 20,515, 1998.

Walker, A. D. M., Excitation of magnetohydrodynamic cavities in the magnetosphere, J. Atm. Sol. Terr. Phys., 60, $1279,1998$.

Walker, A. D. M., J. M. Ruohoniemi, K. B. Baker, and R. A. Greenwald, Spatial and temporal behavior of ULF pulsations observed by the Goose Bay HF radar, J. Geophys. Res., 97, 12,187, 1992.

Wright, A. N., Dispersion and wave coupling in inhomogeneous MHD waveguides, J. Geophys. Res., 99, 159, 1994.

Wright, A. N., and G. J. Rickard, ULF pulsations driven by magnetopause motions: Azimuthal phase characteristics, J. Geophys. Res., 100, 23,703, 1995.

Ziesolleck, C. W. S., and D. R. McDiarmid, Auroral latitude Pc5 field line resonances: Quantized frequencies, spatial characteristics, and diurnal variation, J. Geophys. Res., 99, 5817, 1994.

Ziesolleck, C. W. S., and D. R. McDiarmid, Statistical survey of auroral latitude Pc5 spectral and polarisation characteristics, J. Geophys. Res., 100, 19,299, 1995.

Ziesolleck, C. W. S., F. R. Fenrich, J. C. Samson, and D. R. McDiarmid, Pc5 field line resonances frequencies and structure observed by SuperDARN and CANOPUS, J. Geophys. Res., 103, 11,771, 1998.

I. R. Mann, Department of Physics, University of York, Heslington, York, YO10 5DD, England (e-mail: ian@aurora.york.ac.uk)

A. N. Wright, Mathematical Institute, University of St. Andrews, St.Andrews, Fife, KY16 9SS, Scotland (e-mail: andy@dcs.st-and.ac.uk)

(received March 16, 1999;

revised May 21, 1999;

accepted June 15, 1999.) 